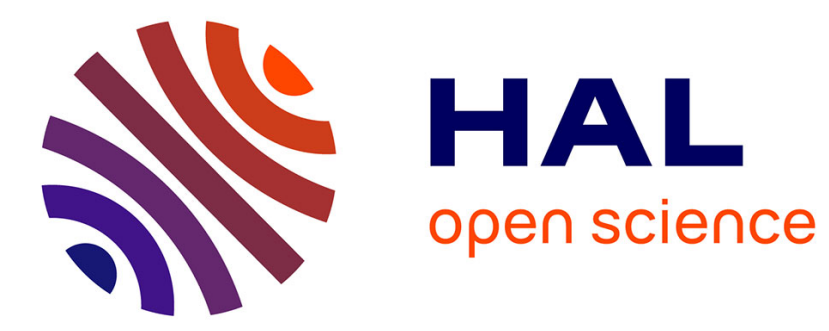

\title{
Valeur énergétique des céréales françaises pour le porc: Synthèse des résultats sur trois années de récolte successives
}

\author{
J. M. Perez
}

\section{- To cite this version:}

J. M. Perez. Valeur énergétique des céréales françaises pour le porc: Synthèse des résultats sur trois années de récolte successives. Productions Animales, 1989, 2 (2), pp.137-143. hal-00895862

\section{HAL Id: hal-00895862 \\ https://hal.science/hal-00895862}

Submitted on 1 Jan 1989

HAL is a multi-disciplinary open access archive for the deposit and dissemination of scientific research documents, whether they are published or not. The documents may come from teaching and research institutions in France or abroad, or from public or private research centers.
L'archive ouverte pluridisciplinaire HAL, est destinée au dépôt et à la diffusion de documents scientifiques de niveau recherche, publiés ou non, émanant des établissements d'enseignement et de recherche français ou étrangers, des laboratoires publics ou privés. 
INRA Prod. Anim., 1989, 2 (2), 137-143

\section{J. M. PEREZ}

INRA Station de Recherches Porcines Saint-Gilles 35590 L'Hermitage

Adresse actuelle : INRA Laboratoire de Recherches sur I'Elevage du Lapin BP 27 - 31326 Castanet-Tolosan Cedex

\section{Valeur énergétique des céréales françaises pour le porc :}

\section{Synthèse des résultats sur trois années de récolte successives}

\begin{abstract}
Avec une consommation annuelle de 4 millions de tonnes, les céréales occupent en France une place prépondérante dans les aliments destinés aux porcins. Leur rôle essentiel étant la fourniture d'énergie, l'intérêt économique des céréales dépend pour une large part de la valeur énergétique qu'on leur attribue. La connaissance de leurs caractéristiques énergétiques moyennes et de leurs variations constitue ainsi une information indispensable pour une meilleure valorisation en alimentation animale.
\end{abstract}

Depuis plusieurs années, un effort important de caractérisation des céréales françaises aux plans analytique et nutritionnel a été entrepris par l'ITCF, l'AGPM et l'ONIC en collaboration avec l'INRA. Mais jusqu'à présent, pour apprécier les variations de la valeur énergétique des céréales, on n'utilisait dans ces enquêtes annuelles que les mesures réalisées sur coqs adultes. Or, les données obtenues chez les volailles ne peuvent être extrapolées au porc, même en valeur relative, sans des études appro-

\section{Résumé}

Quatre-vingt-dix bilans digestifs (six expériences) ont été réalisés sur des porcs mâles castrés de race Large White, à raison de cinq animaux par régime, pour apprécier la valeur énergétique (ED, EM) des céréales françaises au cours de trois années de récolte successives. Les excreta (fèces et urine) ont été collectés en totalité pendant dix jours consécutifs. Les régimes étaient exclusivement à base de céréales $(96 \%)$. Chaque année, six lots de céréales représentatifs de la récolte nationale étaient testés : maïs " Nord France», maïs "Sud France », blé tendre, orge de printemps, orge d'hiver à deux rangs et escourgeon. Les résultats obtenus indiquent de faibles variations entre années et une bonne répétabilité de la valeur énergétique relative des différentes céréales : maïs (111), blé (108), orge de printemps (100), orge d'hiver à deux rangs (100), escourgeon (98). Les valeurs énergétiques moyennes enregistrées avec les six lots de maïs ( $3940 \mathrm{kcal}$ ED $/ \mathrm{kg} \mathrm{MS}$ ) et les trois lots de blé (3830 kcal $\mathrm{ED} / \mathrm{kg} \mathrm{MS}$ ) sont pratiquement identiques aux valeurs de référence (INRA 1984). Pour les neuf lots d'orges, les valeurs trouvées diffèrent seulement de $1 \%$ en moyenne par rapport aux valeurs calculées à partir de leurs teneurs en cellulose brute à l'aide de l'équation proposée antérieurement : ED (kcal $/ \mathrm{kg} \mathrm{MS})=4072 \cdot 110 \mathrm{CB}[\% \mathrm{MS})$. fondies menées parallèlement chez les deux espèces (Leclercq et al 1981, Sibbald et al 1983). En effet, il n'est pas évident que le porc réagisse dans le même sens et avec la même amplitude que les volailles. De plus, le porc est en particulier un gros consommateur d'orges, alors que les volailles n'en utilisent que des quantités réduites. Et à cet égard, le coq n'est peut être pas le modèle idéal pour tester les variations de qualité des céréales destinées essentiellement au porc.

Cette étude a été réalisée dans le cadre des enquêtes précédemment citées avec un triple objectif :

- repréciser les valeurs énergétiques «standard » et la valeur relative des principaux types de céréales pour le porc,

- tester les relations entre les caractéristiques de composition chimique et la valeur énergétique des céréales,

- apprécier la variabilité annuelle de leur valeur alimentaire sur des lots représentatifs de la collecte nationale.

\section{1 / Caractéristiques des céréales}

\section{1 / Constitution des mélanges nationaux représentatifs}

Les lots de céréales utilisés dans cette étude ont été constitués, pendant les campagnes de récolte 1983-84, 1984-85 et 1985-86, par l'ITCF pour les céréales à paille et par l'AGPM pour le 


\section{ENQUETTES ANNUELLES SUR LA QUALTTÉ DES CÉRÉALES}

Les enquêtes ONIC ITCF sur les céréales à paille portent sur 45 départements pour les blés et 30 départements pour les orges, fournissant respectivement $80 \%$ et $70 \%$ de la récolte nationale. L'échantillonnage est établi par l'ONIC au moyen de l'enquête céréalière par sondage, soit à partir de son fichier « livreurs de céréales », soit à partir de photographies aériennes. Le tirage des champs enquêtés et la collecte des échantillons sont assurés par les services régionaux de lOONIC. Quarante échantillons de blé et d'orges sont prélevés dans chaque département dírectement dans les champs à la sortie de la noissonneuse-batteuse. A partir de ces échantillons préalablement nettoyés, sont préparés quatre mélanges nationaux : blé tendre, orge de printemps, orge d'hiver à deux rangs et escourgeon. Chaque mélange national, de $200 \mathrm{~kg}$ environ, est constitué au prorata des surfaces des différentes variétés. Pour les blés, seules les variétés de valeur boulangère médiocre ou irrégulière ont été retenues.

Le champ géographique des enquêtes ONIC-AGPM sur les mais français s'étend sur 19 départements représentant $60 \%$ de la récolte nationale. Les services de l'ONIC ont prélevé à la sortie des séchoirs, à trois reprises pendant la collecte, 10 échantillons de $500 \mathrm{~g}$ de grains chez trois organismes collecteurs pour chacun des départements retenus. Les prélèvements élémentaires ont été mélangés pour obtenir un échantillon représentatif de chaque département enquêté. Trois ou quatre échantillons départementaux ont ensuite été mélangés à parts égales pour constituer chaque mélange régional. Enfin, les échantillons provenant des régions Bassin Parisien et Centre d'une part, et des régions Aquitaine et Midi-Pyrénées d'autre part, ont été mélangés en quantités égales pour obtenir deux lots de $200 \mathrm{~kg}$ de grain représentatifs des maís du « Nord 》 et du « Sud » de la France.

maïs, au cours des enquêtes annuelles sur la qualité des céréales pour l'alimentation animale menées conjointement avec l'ONIC (voir encadré).

\section{2 / Composition chimique et mesures physiques}

Les résultats d'analyse et de mesures physiques obtenus par les laboratoires de l'INRA et de l'ITCF sur les échantillons de céréales sont présentés en fonction de l'année de récolte dans le tableau 1. Les matières azotées sont déterminées conventionnellement à partir de la teneur en azote $(\mathrm{N} \times 6,25)$ dosée selon Kjeldahl. Les matières grasses sont extraites par l'éther diéthylique à l'aide d'un appareil semi-automatique (Soxtec). La cellulose brute correspond au résidu organique de Weende; ce dosage a été répété jusqu'à six fois dans le cas des orges. Les constituants pariétaux selon Van Soest (NDF et ADF) sont déterminés au moyen d'un équipement Fibertec après traitement enzymatique avec une solution d'amylase. L'amidon est dosé par la méthode polarimétrique (Ewers). Le poids spécifique est mesuré au nilémalitre. Le calibrage des orges est effectué à l'aide de l'appareil à secousses du type Steinecker équipé d'un tamis à fentes rectangulaires de $2,5 \mathrm{~mm}$ de largeur.

Pour l'ensemble des analyses, les valeurs mesurées directement sur les mélanges nationaux sont conformes aux valeurs calculées (moyennes pondérées) à partir des déterminations effectuées sur les mélanges régionaux. Ainsi en particulier, les résultats du tableau 1 reflètent parfaitement les variations de composition des céréales enregistrées entre les années de récolte au cours des enquêtes générales [ONIC- ITCF 1983-85, Métayer et Leuillet 1986, ONIC-AGPM 1986).

\section{2 / Etude de digestibilité : modalités expérimentales}

La valeur énergétique et azotée des céréales a été déterminée au cours de six expériences de digestibilité. En raison des dates de récolte plus tardives et des délais de constitution des échantillons moyens représentatifs, les expériences sur les maïs ont été dissociées de celles ayant trait aux céréales à paille. Chaque année, les comparaisons portaient respectivement sur deux lots de maïs et quatre lots de céréales à paille (un échantillon de blé et trois types d'orge).

Au total, 90 bilans digestifs ont été réalisés sur des porcs mâles castrés de race Large White, provenant du troupeau expérimental de l'INRA-St Gilles, à raison de cinq animaux par régime. A l'issue d'une période d'adaptation à la vie en cage à métabolisme d'une semaine environ, les porcs d'un poids vif moyen initial de $30-35 \mathrm{~kg}$ ont été répartis selon les expériences en deux ou quatre lots de cinq animaux homogènes d'après l'âge et le poids, et ont reçu leurs régimes respectifs. Après une phase d'accoutumance de sept jours aux aliments expérimentaux (période de précollecte), on a procédé durant dix jours consécutifs à la collecte totale des excreta (fèces et urine) selon une technique déjà décrite (Perez et al 1984). Au cours de cette période, les niveaux d'alimentation sont constants et la ration est distribuée en deux repas sous forme de farine humide par jour.

Les régimes sont constitués exclusivement de céréales ( $96 \%$ environ). Ils sont simplement rééquilibrés en minéraux, vitamines et acides aminés (lysine, thréonine, tryptophane). La présence d'une seule source de matière organique dans les régimes permet d'estimer directement la valeur énergétique des céréales. 
Tableau 1. Composition chimique (en \% de la matière sèche) et caractéristiques physiques des céréales.

\begin{tabular}{|c|c|c|c|c|c|c|c|}
\hline & $\begin{array}{c}\text { Année } \\
\text { de récolte }\end{array}$ & $\begin{array}{l}\text { Maïs } \\
\text { Nord }\end{array}$ & $\begin{array}{l}\text { Maïs } \\
\text { Sud }\end{array}$ & Blé & $\begin{array}{c}\text { Orge } \\
\text { printemps }\end{array}$ & $\begin{array}{c}\text { Orge } \\
\text { hiver 2R }\end{array}$ & $\begin{array}{c}\text { Orge } \\
\text { hiver 6R }\end{array}$ \\
\hline Matière organique & $\begin{array}{l}83 \\
84 \\
85\end{array}$ & $\begin{array}{l}98,4 \\
98,5 \\
98,6\end{array}$ & $\begin{array}{l}98,5 \\
98,7 \\
98,8\end{array}$ & $\begin{array}{l}97,7 \\
98,1 \\
98,4\end{array}$ & $\begin{array}{l}97,4 \\
97,5 \\
97,6\end{array}$ & $\begin{array}{l}97,1 \\
97,3 \\
97,5\end{array}$ & $\begin{array}{l}97,2 \\
97,4 \\
97,5\end{array}$ \\
\hline $\begin{array}{l}\text { Matières azotées } \\
(\mathrm{N} \times 6,25)\end{array}$ & $\begin{array}{l}83 \\
84 \\
85\end{array}$ & $\begin{array}{r}10,1 \\
9,8 \\
10,1\end{array}$ & $\begin{array}{r}10,3 \\
9,7 \\
9,8\end{array}$ & $\begin{array}{l}12,4 \\
13,0 \\
12,4\end{array}$ & $\begin{array}{l}11,5 \\
11,4 \\
10,8\end{array}$ & $\begin{array}{l}12,0 \\
12,4 \\
11,6\end{array}$ & $\begin{array}{r}9,9 \\
10,6 \\
10,3\end{array}$ \\
\hline Matières grasses & $\begin{array}{l}83 \\
84 \\
85\end{array}$ & $\begin{array}{l}4,6 \\
4,5 \\
4,9\end{array}$ & $\begin{array}{l}4,4 \\
4,3 \\
4,4\end{array}$ & $\begin{array}{l}2,2 \\
1,8 \\
2,2\end{array}$ & $\begin{array}{l}2,1 \\
2,3 \\
1,8\end{array}$ & $\begin{array}{l}2,2 \\
2,1 \\
1,7\end{array}$ & $\begin{array}{l}2,2 \\
2,2 \\
1,8\end{array}$ \\
\hline Cellulose brute & $\begin{array}{l}83 \\
84 \\
85\end{array}$ & $\begin{array}{l}2,7 \\
2,4 \\
2,4\end{array}$ & $\begin{array}{l}2,5 \\
2,4 \\
2,5\end{array}$ & $\begin{array}{l}3,1 \\
2,5 \\
2,6\end{array}$ & $\begin{array}{l}5,0 \\
4,3 \\
4,8\end{array}$ & $\begin{array}{l}5,3 \\
4,6 \\
4,8\end{array}$ & $\begin{array}{l}5,6 \\
5,0 \\
5,3\end{array}$ \\
\hline $\mathrm{NDF}$ & $\begin{array}{l}83 \\
84 \\
85\end{array}$ & $\begin{array}{r}9,8 \\
10,4 \\
10,8\end{array}$ & $\begin{array}{r}9,0 \\
10,4 \\
10,2\end{array}$ & $\begin{array}{l}12,3 \\
12,4 \\
12,3\end{array}$ & $\begin{array}{l}15,8 \\
14,8 \\
13,5\end{array}$ & $\begin{array}{l}16,2 \\
15,1 \\
14,4\end{array}$ & $\begin{array}{l}16,7 \\
15,5 \\
15,5\end{array}$ \\
\hline $\mathrm{ADF}$ & $\begin{array}{l}83 \\
84 \\
85\end{array}$ & $\begin{array}{l}3,7 \\
2,7 \\
2,4\end{array}$ & $\begin{array}{l}3,1 \\
2,7 \\
2,5\end{array}$ & $\begin{array}{l}4,4 \\
3,7 \\
3,7\end{array}$ & $\begin{array}{l}6,0 \\
4,8 \\
5,8\end{array}$ & $\begin{array}{l}6,1 \\
5,6 \\
5,8\end{array}$ & $\begin{array}{l}6,5 \\
5,6 \\
6,0\end{array}$ \\
\hline Amidon Ewers & $\begin{array}{l}83 \\
84 \\
85\end{array}$ & $\begin{array}{l}72,4 \\
73,3 \\
72,9\end{array}$ & $\begin{array}{l}72,6 \\
73,3 \\
73,2\end{array}$ & $\begin{array}{l}70,8 \\
68,6 \\
69,0\end{array}$ & $\begin{array}{c}60,7 \\
61,1 \\
-\end{array}$ & $\begin{array}{c}62,2 \\
58,3 \\
-\end{array}$ & $\begin{array}{c}60,3 \\
59,2 \\
-\end{array}$ \\
\hline $\begin{array}{l}\text { Poids spécifique } \\
(\mathrm{kg} / \mathrm{hl})\end{array}$ & $\begin{array}{l}83 \\
84 \\
85\end{array}$ & $\begin{array}{l}- \\
- \\
-\end{array}$ & $\begin{array}{l}- \\
- \\
-\end{array}$ & $\begin{array}{l}76,5 \\
78,9 \\
78,1\end{array}$ & $\begin{array}{l}67,1 \\
70,0 \\
70,3\end{array}$ & $\begin{array}{l}66,9 \\
68,3 \\
68,5\end{array}$ & $\begin{array}{l}65,3 \\
67,3 \\
67,2\end{array}$ \\
\hline Calibrage $(\%<2,5 \mathrm{~mm})$ & $\begin{array}{l}83 \\
84 \\
85\end{array}$ & $\begin{array}{l}- \\
- \\
-\end{array}$ & $\begin{array}{l}- \\
- \\
-\end{array}$ & $\begin{array}{l}- \\
- \\
-\end{array}$ & $\begin{array}{l}20,4 \\
13,6 \\
13,2\end{array}$ & $\begin{array}{r}16,5 \\
5,6 \\
6,8\end{array}$ & $\begin{array}{l}31,3 \\
14,6 \\
18,5\end{array}$ \\
\hline
\end{tabular}

Tableau 2. Valeur énergétique des céréales.

\begin{tabular}{|c|c|c|c|c|c|c|c|}
\hline & $\begin{array}{c}\text { Année } \\
\text { de récolte }\end{array}$ & $\begin{array}{l}\text { Maïs } \\
\text { Nord }\end{array}$ & $\begin{array}{l}\text { Maïs } \\
\text { Sud }\end{array}$ & Blé & $\begin{array}{c}\text { Orge } \\
\text { printemps }\end{array}$ & $\begin{array}{c}\text { Orge } \\
\text { hiver 2R }\end{array}$ & $\begin{array}{c}\text { Orge } \\
\text { hiver 6R }\end{array}$ \\
\hline Energie Brute (1) & $\begin{array}{l}83 \\
84 \\
85 \\
\end{array}$ & $\begin{array}{l}4513 \\
4530 \\
4462 \\
\end{array}$ & $\begin{array}{l}4479 \\
4459 \\
4433 \\
\end{array}$ & $\begin{array}{l}4460 \\
4441 \\
4452 \\
\end{array}$ & $\begin{array}{l}4441 \\
4458 \\
4374 \\
\end{array}$ & $\begin{array}{l}4431 \\
4454 \\
4416 \\
\end{array}$ & $\begin{array}{l}4455 \\
4424 \\
4392 \\
\end{array}$ \\
\hline Energie Digestible (2) & $\begin{array}{l}83 \\
84 \\
85\end{array}$ & $\begin{array}{r}3948 \\
\pm \quad 16 \\
3954 \\
\pm \quad 17 \\
3952 \\
\pm \quad 10\end{array}$ & $\begin{array}{r}3955 \\
\pm \quad 13 \\
3912 \\
\pm \quad 27 \\
3918 \\
\pm \quad 8\end{array}$ & 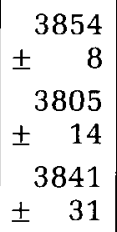 & $\begin{array}{r}3577 \\
\pm \quad 21 \\
3512 \\
\pm \quad 18 \\
3546 \\
\pm \quad 16\end{array}$ & $\begin{array}{r}3520 \\
\pm \quad 24 \\
3518 \\
\pm \quad 6 \\
3541 \\
\pm \quad 18\end{array}$ & $\begin{array}{r}3498 \\
\pm \quad 13 \\
3467 \\
\pm \quad 6 \\
3489 \\
\pm \quad 4\end{array}$ \\
\hline CUDa Energie (\%) & $\begin{array}{l}83 \\
84 \\
85\end{array}$ & $\begin{array}{l}87,5 \\
87,3 \\
88,6\end{array}$ & $\begin{array}{l}88,3 \\
87,7 \\
88,4\end{array}$ & $\begin{array}{l}86,4 \\
85,7 \\
86,3\end{array}$ & $\begin{array}{l}80,5 \\
78,6 \\
81,5\end{array}$ & $\begin{array}{l}79,4 \\
79,0 \\
80,1\end{array}$ & $\begin{array}{l}78,5 \\
78,4 \\
79,4\end{array}$ \\
\hline $\begin{array}{l}\text { Energie } \\
\text { Métabolisable } \\
\text { apparente }\end{array}$ & $\begin{array}{l}83 \\
84 \\
85\end{array}$ & $\begin{array}{l}3856 \\
3860 \\
3854\end{array}$ & $\begin{array}{l}3865 \\
3823 \\
3825\end{array}$ & $\begin{array}{l}3752 \\
3669 \\
3715\end{array}$ & $\begin{array}{l}3493 \\
3412 \\
3453\end{array}$ & $\begin{array}{l}3438 \\
3408 \\
3437\end{array}$ & $\begin{array}{l}3422 \\
3372 \\
3397\end{array}$ \\
\hline $\mathrm{EMa} / \mathrm{ED}(\%)$ & $\begin{array}{l}83 \\
84 \\
85\end{array}$ & $\begin{array}{l}97,7 \\
97,6 \\
97,5\end{array}$ & $\begin{array}{l}97,7 \\
97,7 \\
97,6\end{array}$ & $\begin{array}{l}97,4 \\
96,4 \\
96,7\end{array}$ & $\begin{array}{l}97,7 \\
97,1 \\
97,4\end{array}$ & $\begin{array}{l}97,7 \\
96,9 \\
97,1\end{array}$ & $\begin{array}{l}97,8 \\
97,3 \\
97,4\end{array}$ \\
\hline
\end{tabular}

Les valeurs énergétiques ont été mesurées au cours de trois campagnes de récolte successives sur des échantillons représentatifs de la collecte nationale de céréales. 


\section{3 / Résultats et discussion}

A partir des résultats de digestibilité des régimes, nous avons déduit les valeurs nutritives moyennes des céréales, qui figurent dans le tableau 2. D’une façon générale, on constate que la précision de l'estimation des valeurs énergétiques est excellente, puisque sur l'ensemble des expériences les erreurs-types oscillent entre 4 et $31 \mathrm{kcal}$ sur la base de la matière sèche, ce qui correspond à des coefficients de variation (CV) inférieurs à $1 \%$ en moyenne $(0,2$ à 1,8\% de valeurs extrêmes). En conformité avec les données classiques sur les céréales (Perez et al 1978), on vérifie aussi sur le tableau 2 que l'énergie métabolisable apparente (EMa) représente une proportion élevée et sensiblement constante de l'énergie digestible $(97,4$

Figure 1. Valeurs $\pm 0,4 \%)$.

energétiques des céréales.

Energie digestible ( $\mathrm{kcal} / \mathrm{kg} \mathrm{MS})$

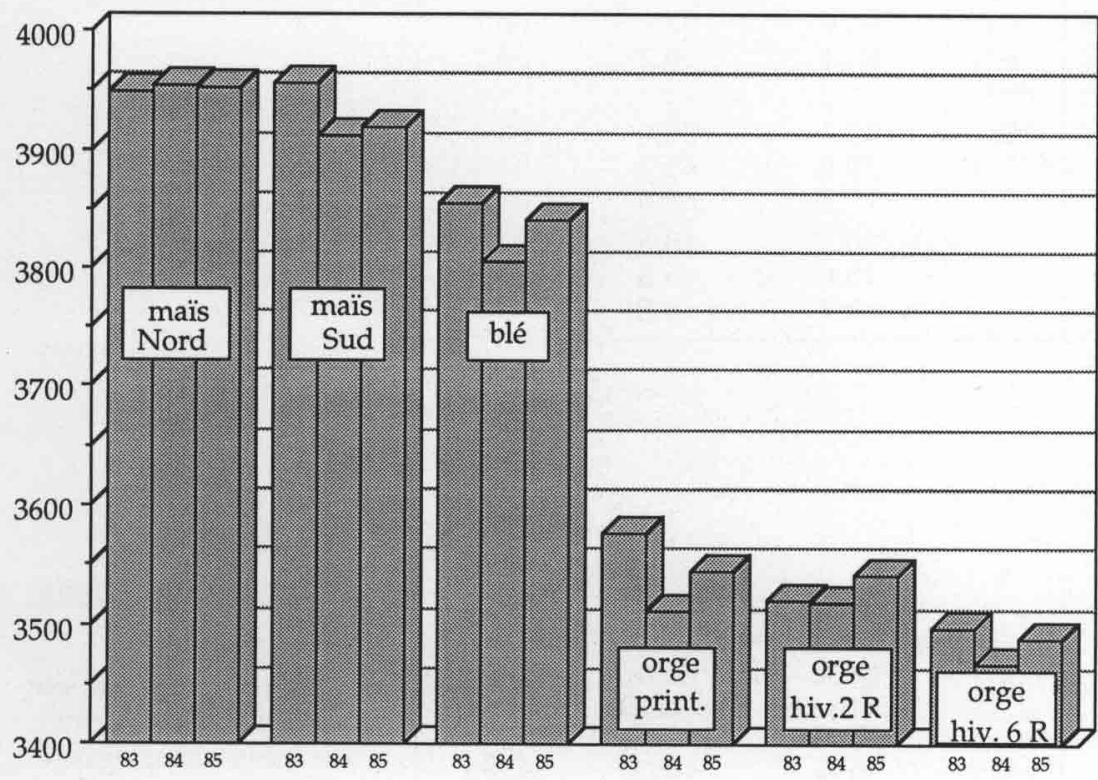

Les effets de l'année de récolte sur les variations de la valeur énergétique des céréales sont analysés dans la figure 1 . Sur la période considérée (1983-85), les variations intra-type sont faibles puisque les coefficients de variation s'élèvent en moyenne à $0,5 \%$ et ne dépassent en aucun cas $1 \%$, quelle que soit la nature de la céréale. énergétique est répétable d'une année

sur l'autre, la valeur de chaque type variant peu selon l'année.
De la même façon, les écarts de valeur énergétique entre les différents types de céréales sont répétables d'une année sur l'autre. Ainsi, les valeurs en énergie digestible du maïs et du blé sont supérieures en moyenne respectivement de 11 et $8 \%$ par rapport à la valeur de l'orge de printemps prise comme référence (tableau 3), ce qui corrobore nos estimations antérieures sur la valeur relative des principales céréales (Perez et al 1978). Parmi les orges, les types hiver à deux rangs ne diffèrent pas de leurs homologues de printemps, alors que les escourgeons (hiver à six rangs) présentent des valeurs énergétiques inférieures d'environ $2 \%$. Cette faible différence (50 kcal ED $/ \mathrm{kg} \mathrm{MS}$ ), observée dans cette étude entre les escourgeons et les orges à deux rangs par rapport aux données classiques, s'explique par un resserrement progressif des écarts de teneurs en cellulose brute entre les différents types d'orge sous l'influence des évolutions variétales (Perez et Leuillet 1986). Ainsi, pour les lots testés sur la période 1983-85, l'écart moyen de teneur en cellulose brute entre les escourgeons et les orges à deux rangs est de 0,5 point seulement, alors que nous l'avions fixé à 1,4 point sur la base des enquêtes antérieures lors de la constitution des tables INRA (1984), ce qui se traduisait par une différence de valeur énergétique de $4,5 \%$ (150 kcal ED/kg MS) entre ces deux grandes catégories d'orge.

Les mesures d'énergie métabolisable réalisées parallèlement chez les volailles (Lessire, communication personnelle) sur les mêmes lots de blé et d'orges montrent que les valeurs énergétiques relatives des céréales à paille sont assez proches chez les deux espèces animales, et ceci de manière répétable au cours des trois années de récolte. Toutefois, on observe un écart un peu plus marqué entre types d'orges extrêmes chez les volailles et une valeur relative plus élevée du blé chez le porc.

Dans le tableau 4, nous avons comparé nos résultats expérimentaux avec les données théoriques. Pour le maïs et le blé, nous avons retenu comme références les valeurs moyennes des tables INRA (1984). Pour les orges, nous avons calculé les valeurs énergétiques théoriques à partir de leurs teneurs en cellulose brute à l'aide de l'équation proposée antérieurement (Perez et al 1980).

Pour le maïs, les valeurs ED enregistrées sont voisines pour les deux catégories (Nord et Sud) et sont conformes voire strictement identiques

Tableau 3. Effet de l'année de récolte sur la valeur énergétique relative des différents types de céréales (indice 100 pour l'orge de printemps).

\begin{tabular}{|l|cc|c|rrc|}
\hline & $\begin{array}{c}\text { Maïs } \\
\text { Nord }\end{array}$ & $\begin{array}{c}\text { Maïs } \\
\text { Sud }\end{array}$ & Blé & $\begin{array}{c}\text { Orge } \\
\text { printemps }\end{array}$ & $\begin{array}{c}\text { Orge } \\
\text { hiver 2R }\end{array}$ & $\begin{array}{c}\text { Orge } \\
\text { hiver 6R }\end{array}$ \\
\hline Récolte 83 & 110,4 & $\mathbf{1 1 0 , 6}$ & 107,7 & 100 & 98,4 & 97,8 \\
Récolte 84 & 112,6 & 111,4 & 108,3 & 100 & 100,2 & 98,7 \\
Récolte 85 & 111,4 & 110,5 & 108,3 & $\mathbf{1 0 0}$ & 99,9 & 98,4 \\
\hline Moyenne des 3 années & 111,5 & 110,8 & 108,1 & $\mathbf{1 0 0}$ & 99,5 & 98,3 \\
\hline
\end{tabular}


Tableau 4. Comparaison des résultats de valeur énergétique avec les données théoriques (Energie Digestible ED en $\mathrm{kcal} / \mathrm{kg} \mathrm{MS}$ ).

\begin{tabular}{|c|c|c|c|c|c|c|}
\hline & $\begin{array}{l}\text { Maïs } \\
\text { Nord }\end{array}$ & $\begin{array}{l}\text { Maïs } \\
\text { Sud }\end{array}$ & Blé & $\begin{array}{c}\text { Orge } \\
\text { printemps }\end{array}$ & $\begin{array}{c}\text { Orge } \\
\text { hiver 2R }\end{array}$ & $\begin{array}{c}\text { Orge } \\
\text { hiver 6R }\end{array}$ \\
\hline \multicolumn{7}{|l|}{ Récolte 83} \\
\hline ED mesurée & 3948 & 3955 & 3854 & 3577 & 3520 & 3498 \\
\hline ED théorique (1) & 3950 & 3950 & 3850 & 3520 & 3490 & 3456 \\
\hline Ecart en \% (2) & 0 & 0 & 0 & $+1,6$ & $+0,9$ & $+1,2$ \\
\hline \multicolumn{7}{|l|}{ Récolte 84} \\
\hline ED mesurée & 3954 & 3912 & 3805 & 3512 & 3518 & 3467 \\
\hline ED théorique & 3950 & 3950 & 3850 & 3596 & 3567 & 3520 \\
\hline Ecart en \% & 0 & $-1,0$ & $-1,2$ & $-2,3$ & $-1,4$ & $-1,5$ \\
\hline \multicolumn{7}{|l|}{ Récolte 85} \\
\hline ED mesurée & 3952 & 3918 & 3841 & 3546 & 3541 & 3489 \\
\hline ED théorique & 3950 & 3950 & 3850 & 3544 & 3544 & 3489 \\
\hline Ecart en \% & 0 & $-0,8$ & $-0,2$ & 0 & 0 & $\mathbf{0}$ \\
\hline
\end{tabular}

(1) Pour le maïs et le blé : données moyennes des tables INRA (1984)

Pour les orges, FD calculée avec l'équation (Perez et al 1980): ED = 4072-110 CB (\% MS).

(2) ED monesurée/ED théorique.

à la valeur standard (INRA 1984). On constate néanmoins un léger écart $(+1 \%)$ en 1984 et 1985 en faveur des lots en provenance du nord de la France. Cet écart, inférieur à $40 \mathrm{kcal}$, apparaît statistiquement significatif $(\mathrm{P}<0,05)$ en 1985, en raison de la précision de la méthode directe de détermination de la valeur énergétique et d'une variabilité de réponse entre animaux particulièrement faible au cours de cette campagne: le coefficient de variation moyen pour ce critère est en effet de $0,5 \%$ seulement. Cette différence de contenu énergétique entre les deux catégories de maïs peut s'ex- pliquer par une teneur un peu plus élevée en matières grasses ( $+0,3$ point, 4,7 vs $4,4 \%$ ) et corrélativement en énergie brute $(+1 \%, 4502$ vs $4457 \mathrm{kcal}$ ) des maïs de type corné-denté en provenance du nord de la France par rapport à ceux du sud de la France (grains dentés). En revanche, l'utilisation digestive de l'énergie (CUD E) ne varie pas selon le lot de maïs et est identique à la valeur des tables INRA ( $88 \%$ ). Les résultats obtenus dans cette étude confirment, sur des mélanges représentatifs de la collecte nationale, la faible variabilité de la valeur énergétique des maïs français observée précé-

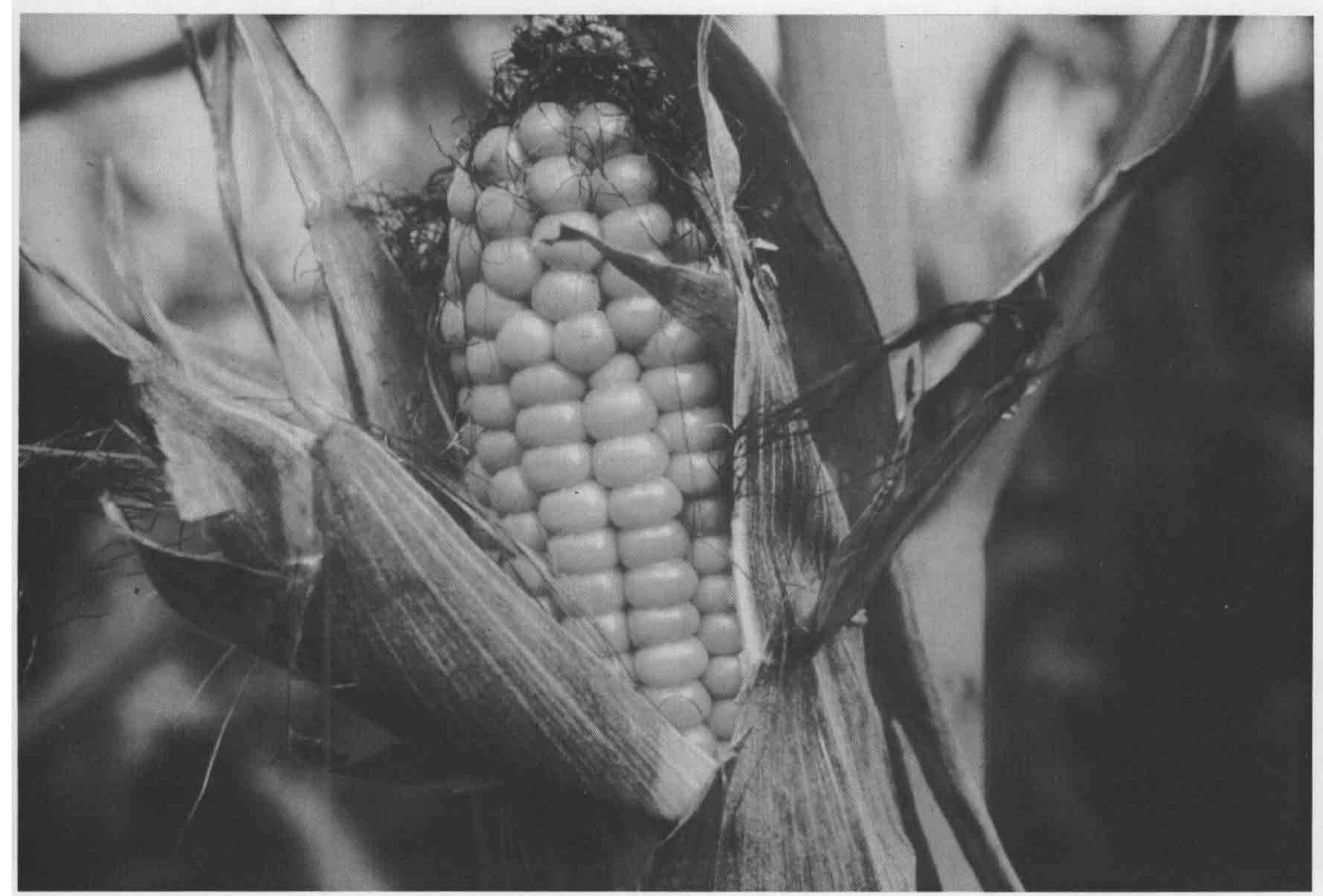

Les résultats sont similaires aux valeurs des tables INRA 1984

(maïs, blé) ou aux données théoriques calculées par régression (orges). 


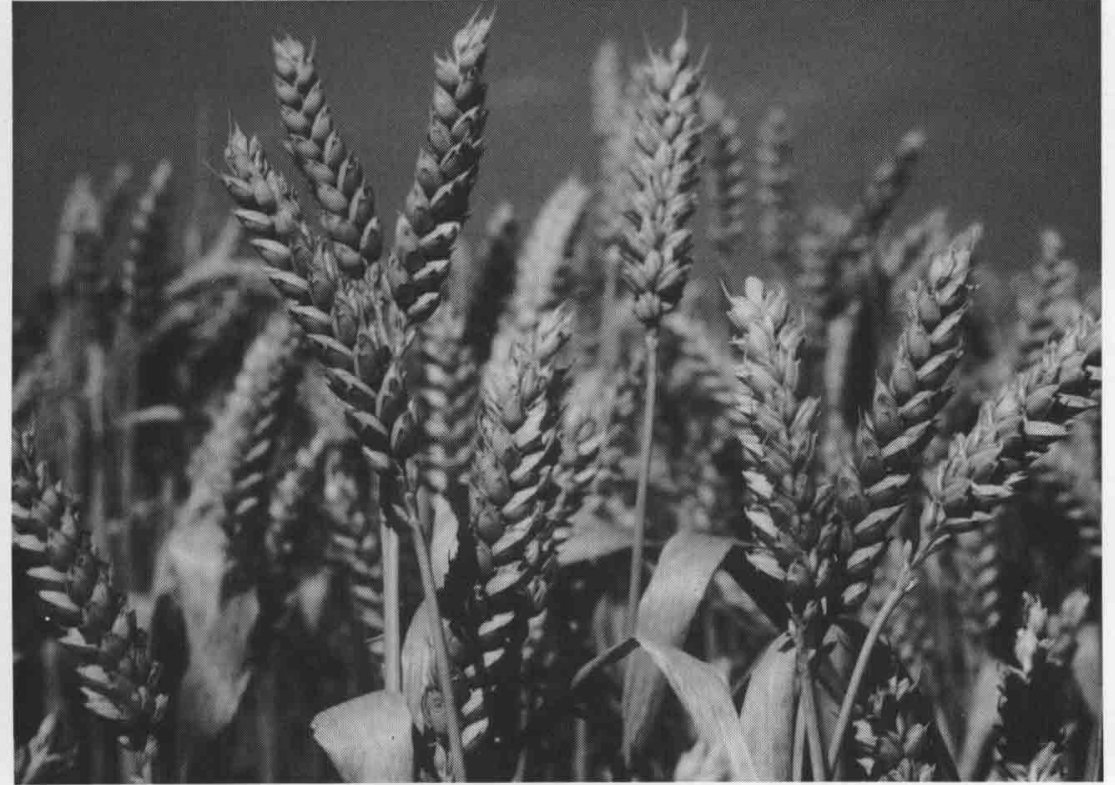

Epis de blé mûr. demment dans notre laboratoire sur des lots ponctuels : $3935 \mathrm{kcal}$ ( $\pm 1 \%$ ) (Henry 1968, Perez et Aumaitre 1979, Bourdon et Baudet 1979, Bourdon et Perez 1982, Bourdon et al 1982. Perez et Bourdon 1984).

A titre de comparaison, on aboutit à une même valeur énergétique moyenne (3950 kcal $\mathrm{EL} / \mathrm{kg} \mathrm{MS}$ ) sur des maïs de diverses origines à partir de l'ensemble des données bibliographiques étrangères (30 déterminations). Si dans ce cas, la dispersion des résultats est plus grande ( $\pm 3 \%$ ), cela résulte probablement beaucoup plus de problèmes liés aux méthodes d'estimation de la valeur énergétique qu'à la variabilité des caractéristiques intrinsèques de cette céréale.

Pour le blé, les valeurs ED obtenues sont également très proches des données théoriques, puisque seul l'échantillon de 1984 s'écarte légèrement $(-1,2 \%)$ de la valeur standard (3 850 kcal). Cette légère baisse de la valeur énergétique peut difficilement s'expliquer par des variations de composition chimique. Elle n'était pas non plus prévisible en fonction des bonnes caractéristiques physiques observées généralement au cours de cette campagne (Métayer et Leuillet 1986) et confirmées par les mesures effectuées sur les mélanges nationaux: poids spécifique élevé $(78,9 \mathrm{~kg} / \mathrm{hl})$ et poids de 1000 grains exceptionnel $(38,6$ contre 32,5 et $36,2 \mathrm{~g}$ MS respectivement en 1983 et 1985). Quoiqu'il en soit, les variations de contenu énergétique enregistrées sont minimes et corroborent, comme pour le maïs, les mesures effectuées dans notre laboratoire sur des lots ponctuels :
$3820 \mathrm{kcal} \mathrm{(} \pm 1 \%$ ) (Henry 1968, Bourdon et al 1980, Bourdon et Perez 1982, Perez et al 1986). Nos résultats sont également en accord avec les valeurs ED moyennes ( 3870 et 3835 kcal) obtenues respectivement sur huit échantillons de blés autraliens et de blés anglais par Batterham et al (1980) et Wiseman et al (1982). De grandes variations dans la valeur énergétique des blés ne semblent s'observer qu'à la suite de conditions climatiques difficiles (Anderson et Bell 1983)

Pour les orges, bien que la gamme de teneurs en cellulose brute soit étroite, les écarts entre les valeurs mesurées et les valeurs théoriques calculées par régression (tableau 4) sont très faibles ( $1 \%$ en moyenne) et à la limite de la précision expérimentale. En 1983, l'équation de prédiction utilisée tend à sous-estimer légèrement la valeur énergétique des orges, alors que l'inverse se produit l'année suivante. En 1985, il y a concordance parfaite entre les valeurs mesurées et les valeurs prédites. Au total, notre équation de prédiction, établie sur des échantillons d'orges de la récolte 1978, semble avoir une portée assez générale, comme l'ont confirmé d'autres mesures effectuées depuis dans notre laboratoire et également les résultats de Bell et al (1983) sur des orges reconstituées à partir de proportions variables d'enveloppes fibreuses.

\section{Conclusion}

Les résultats obtenus dans cette étude, au cours de trois campagnes de récolte successives sur des échantillons moyens représentatifs de la collecte nationale, indiquent de faibles variations entre années et une bonne répétabilité de la valeur énergétique relative des différentes céréales pour le porc: maïs (111), blé (108), orge de printemps (100), orge d'hiver à deux rangs (100), escourgeon (98). Les valeurs énergétiques moyennes enregistrées avec les six lots de maïs (3 $940 \mathrm{kcal} \mathrm{ED/kg} \mathrm{MS}$ ) et les trois lots de blé ( $3830 \mathrm{kcal} \mathrm{ED} / \mathrm{kg} \mathrm{MS}$ ) sont pratiquement identiques aux valeurs de référence (INRA 1984). Pour les neuf lots d'orges, les valeurs trouvées diffèrent seulement de $1 \%$ en moyenne par rapport aux valeurs calculées par régression à partir de leurs teneurs en cellulose brute (Perez et al 1980).

\section{Remerciements}

Ces travaux ont fait l'objet de conventions de recherche et ont bénéficié du soutien financier de l'ONIC (Office National Interprofessionnel des Céréales), de l'ITCF (Institut Technique des Céréales et des Fourrages) et de l'AGPM (Association Générale des Producteurs de Maïs). 


\section{Références bibliographiques}

ANDERSON D.M., BELL J.M., 1983. The digestibility by pigs of dry matter, energy, protein and amino acids in wheat cultivars. II/ fifteen cultivars grown in two years, compared with Bonanza and Fergus barlevs, and $3 \mathrm{CW}$ grade hard red spring wheat. Can. J. Plant Sci., 63, 393406.

BATTERHAM E.S., LEWIS C.E., LOWE R.F. MCMILLAN C.I., 1980. Digestible energy content of cereals and wheat by-products for growing pigs. Anim. Prod., 31, 259-271.

BELL J.M., SHIRES A., KEITI I M.O., 1983. Fffect of hull and protein contents of barley on protein and energy digestibility and feeding value for pigs. Can. J. Anim. Sci.. 63, 201-211.

BOURDON D., PEREZ J.M., HENRY Y., CALMES R., 1980. Valeur énergétique et azotée d'un concentré de protéines de luzerne, le "PX1" et utilisation par le porc en croissance-finition. Journées Rech. Porcine en France, 12, $227-244$.

BOURDON D.. QUERE P. BAUDFT I.I., 1982. Valeur énergétique et azotée chez le porc d'un tourteau de colza français à faible teneur en glucosinolates et dépelliculé. Journées Rech. Porcine en France, 14, 297-306.

BOURDON D., BAUDET' J.J., 1979. Valeur énergétique el azotée de tourteaux de colza à faible teneur en glucosinolates ou dépelliculé et d'un tourteau de tournesol chez le porc en croissance. Journées Rech. Porcine en France, 11. $283-290$.

BOURDON D., PERFZ J.M., 1982. Valeur nutritive du triticale pour le porc: comparaison avec le blé, le seigle t: un maïs riche en protéines. Journées Rech. Porcine en France, 14, 223- 230.

HENRY Y. 1968. Utilisation comparée des céréales comme seuls aliments du porc pendant la période de finition. Ann. Zootech., 17, 183-197.

INRA, 1984. L'alimentation des animaux monogastriques : porc, lapin, voláilles. INRA éd., Paris, $282 \mathrm{p}$.

LECLERCQ B., PITON P., GUY G., PEREZ I.M., BOURDON D., 1981. Comparaison de la valeur énergétique des matières premières entre différentes espèces animales (ral, porc, volailles] : cas des orges. Rev. Aliment. Anim., n" 349, 35-38.

METAYER J.P.. LEUILLE:T M. 1986. Bilan des enquêtes annuelles sur blé et orges. ITCF éd., Paris.

ONIC- $\Lambda$ GPM, 1986. La qualité du maïs français: trois années d'enquête. AGPM éd., Pau.

ONIC-I'TCF, 1983-85. Qualité des blés pour l'alimentation animale. I"TCF éd., Paris (publication annuelle).

ONIC-ITCCF, 1983-85. Qualité des orges pour l'alimentation animale. ITCF éd.. Paris (publication annuelle).

PEREZ J.M., AUMAITRE A., 1979. Waxy versus regular maize : energy value for growing pigs and utilization in piglet diets. Anim. Feed Sci. Technol., 4, 109-115.

PEREZ I.M., BOURDON J., 1984. Prévision de la valeur énergélique et azotée des sorghos à partir de leurs teneurs en tanins. Journées Rech. Porcine en France, 16 293-300.

PEREZ, J.M., LFUILLET' M., 1986. Composition et valeur nutritive des céréales. Perspectives Agricoles, $\mathrm{n}^{0}$ 105, 5661.

PEREZ J.M., BOURDON I)., IENRRY Y., 1978. Les céréales dans l'alimentation du porc. Bull. T'ech. Inf. Minist. Agric., n" 331, 335-361.

PEREZ I.M.. RAMOELINTSALAMA B., BOURDON D. 1980. Prévision de la valeur énergétique de l'orge pour le porc à partir des teneurs en constituants membranaires. Journées Rech. Porcine en France, 12, 273-285.

PFRFZ I.M.. RAMIIONF R., HENRY Y., 1984. Prédiction de la valeur énergétique des aliments composés destinés au Porc: étude expérimentale. INRA éd., Versailles, 95 p. PEREZ J.M., BOURDON D., BAUDFT J.J., EVRARD J., 1986. Prévision de la valeur énergétique des tourteaux de tournesol à partir de leurs leneurs en constiluants pariétaux. Journées Rech. Porcine en France, 18, 35-46.

SIBBALD I.R.. ELLIOT J.I., COTE M., 1983. Comparison of bioavialable energy values of diets measured with poultry and swine. Anim. Feed Sci. Technol., 8, 191-196. WISFM AN J., COLE D.J.A., LEWIS D., 1982. Energy values in pig nutrition. 3 / The digestible and metabolizable energy content of barlev, wheat, maize, oats and rye J. Agric. Sci., Camb., 98, 89-97.

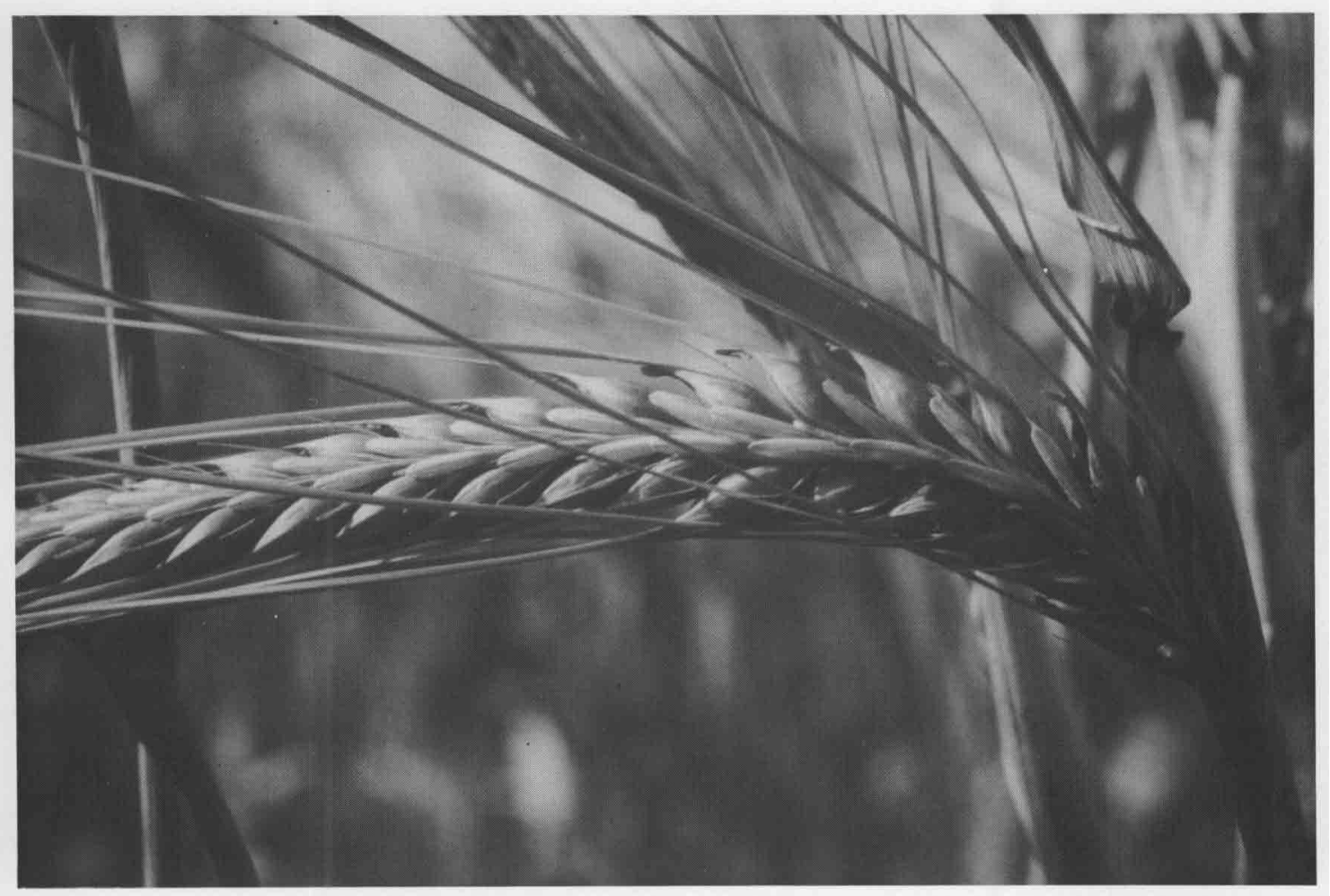

